\title{
Interpretation of Meme Conversations Using Multimodality Analysis
}

\author{
Lutfi Gumilang \& Juanda, UNIKOM-Indonesia
}

\begin{abstract}
The research entitled "Interpretation of Meme Conversations Using Multimodality Analysis". The research has purposes to analyze the meaning of the meme at 9GAG Official Instagram account and also to describe the steps can be taken by use a proper English grammatical rules for the remedial in improving the meaning interpretation. The research used the theories of language errors by Kress and Leeuwen. The method of this research is a descriptive-analytic method. The analysis is based on an investigation of an event, essay, or act for know the real situation. This research is done by describing the data and information obtained, the writer used scientific journals, the sources are downloaded from the internet, as well as through visual media such as visual image from the 9GAG Instagram account and at the final stage will be the conclusion of the analysis results. There are found that meme can be used to deliver a certain message, as a medium for a massive communication and also as an entertainment. The suggestions of the research are it would be better for the reader to understand the meaning of the meme, it will helps the reader to be more completely understand the message or even the entertainment of the meme. Therefore the reader will be able to acknowledge the information.
\end{abstract}

Keywords: meme; multimodality; Instagram

\section{Introduction}

A meme is an idea, behavior or style that spreads from one person to another in a culture. A meme is a new word by Richard Dawkins. According to the theory of Dawkins, ideas, theories, applications, customs, songs, dances, and the mood is an example of a meme. Meme has opened a new way to combine various elements such as creativity, art, message, and humor into the Internet culture.

Nowadays, to express feelings, represent the condition or situation and criticize certain phenomena can be contained in meme, as meme has been regarded as a new medium that is not only used as a medium to deliver the message, but also used as a medium of entertainment. At this present time the spread of meme increasingly widespread. This is because a meme can be easily spread through social media such as Twitter, Instagram, Facebook, Line, and others. Many accounts in social media is often upload meme with ideas or a different topic. One of the media who act as promoters widely meme is 9 GAG Instagram account. 9GAG has also become a social media promoters whose role is to make memes, upload it and popularized it, therefore the meme uploaded by 9 GAG account is able to be accepted by the worldwide society.

Based on the writer's interest would be a study on the analysis of the meme using Multimodality Analysis, This research is very worthy of study because social media is a proof of age has been advanced with the development of technology. Society can issue the imagination, feeling through a drawing and writing. And can convey the message implied by the use of meme. Meme is a habit and one unit of cultural information such as, the idea, the idea of someone in conveying the message. Meme is a testament to the development of science and technology that people can express their intentions quickly. With the development of society can convey meaning and purpose through meme.

Therefore, this study focuses on the interpretation of the meaning of the memes in the official Instagram account belonging to 9GAG. The interpretation of the meaning of that meme will be analyzed by using Multimodality Analysis. In particular, the research questions of this research are as follows: (1) What kind of Meme that is capable of being a medium to deliver the message to the wider community, according to 9GAG Instagram account?, and (2) What is the interpretation of the meme analyzed by using Multimodality Analysis, according to 9GAG Instagram account? 


\section{Literature Review}

Kress and van Leeuwen (2006) said that: "The use of several semiotic modes in the design of a specific product, or event semiotic simultaneously, and by the way this particular coupled modes for-Strengthen, complement, or be in a certain order." In the context of analyzing a text, multimodality analysis can be understood as an analytical procedure that combines aspects and linguistic analysis steps such as Semantics and Discourse. Multimodality analysis combines two aspects or two modes in the process of analyzing a text to get the meaning. The two modes in the form of verbal mode and image mode, which can be used to convey the message to be conveyed, and the meanings, representation of perspective, as well as the meaning of the visual image displayed.

Verbal mode is an aspect in the form of a word, sentence, phrase or speech that is written or imprinted, while the picture mode as particular forms shown. Both of these modes included in combining visual communication such as writing, art, typography, drawing, graphic design, illustration, and color in its delivery. Therefore, Multimodality Analysis method is considered as the most effective method for analyzing the meaning which is interpreted by the meme or visual images are displayed. Since the method of Multimodality Analysis combines both modes in the analysis process so as to produce a clearer meaning and be able to convey the message of the visual image.

According to the theory of Iedema (2003: 29) that Multimodality Analysis can also be defined as "a technical term that aims to show that the meaning of what we do during this Utilizes various semiotic," which means that the method of multimodality is a rule, which technically could be used to show the meaning of an utterance or semiotic expression. While Chen (2010: 485 ) interprets multimodality as "Understanding how verbal and visual semiotic resources can be used to Realize the type and level of dialogic engagement." which means that the method of multimodality is an understanding of verbal language and can be used also to understand the type or types and levels of a dialogue. Among the difference mode used by the public simultaneously in a text to communicate their messages, it allows the combination of sentences of certain verbal and visual images

Meme is a term that was first put forward and popularized by Richard Dawkins. Meme is a good spread of the phenomenon in the form of words, art, culture or images. Over time, the meme itself has undergone various developments; there is a form of words, pictures, and even a combination of both.

Therefore, according to the theory, we can conclude that Multimodality Analysis is a method for analyzing text and visual images that combine the use of various visual communication such as writing, language, images, gesture, icons, art, typography, drawing, graphic design, illustration, and color noise even in its delivery. Where the results of analysis of the forms of communication, is able to affect the meaning and content of the message to be conveyed.

\section{Research Method}

The research object is the meme that have been downloaded by the writer of the official Instagram account belonging to $9 \mathrm{GAG}$ which is being posted on 2018, that has been already verified. Here, researchers are looking for the facts about what is contained meaning of meme, which its form is a messaging or chat. The research method used by the writer in this study is using qualitative research methods. The purpose of using a qualitative approach is that the writer would be able to describe the reality behind the phenomena associated with the analysis of the meaning of meme.

The data collection technique is a method used to collect the data itself. The data will be analyzed in this study are some of the visual image or Meme downloaded from official Instagram account 9GAG. Which is the meme was uploaded by 9GAG account in 2018. The stages of data collection are as follows: 
i) Selecting existing Meme from the official 9 GAG instagram account. The Memes chosen by the writer are the memes in a form of Chat.

ii) Downloading Meme who have chosen to serve the data and then analyzed by the writer.

After all the data has been collected, then the data will be analyzed by the author. The stages of the process of data analysis are as follows:

i) analyze the various elements that exist in the meme in the form of: language, text, typography, color, expression, character, graphic design and illustration or words that exist in meme with other aspects related.

ii) interpret the meaning of what is produced from the process of analysis, uses the method of Multimodality Analysis and draws conclusions from the meme.

\section{Data Analysis}

The writer makes analysis using Multimodality Analysis by Kress and van Leeuwen (2006) to interpret the meme. In the context of text analysis, multimodality can be understood as an analytical procedure that combines aspects and linguistic analysis steps such as Semantics and Discourse. With aspects of the analysis to understand the picture, when text is analyzed using two modes, verbal and images, which can be used to convey the message to be conveyed, and the meanings, representation of perspective, and meaning of visual images that will be offered. Including incorporating visual communication, writing, art, typography, drawing, graphic design, illustration, and color in its delivery.

Multimodality method is considered as the most effective method to analyze the meaning interpreted by the meme or visual images are displayed. The following are the results of research regarding an image that contains a conversation between participants involved. Images are a snapshot or a screenshot of a conversation. The data obtained by downloading the images that have been selected, the data comes from official Instragram account belonging to 9GAG. The results of the data analysis are as follows:

\section{Picture 1. The Conversation about A Carrot Ring}

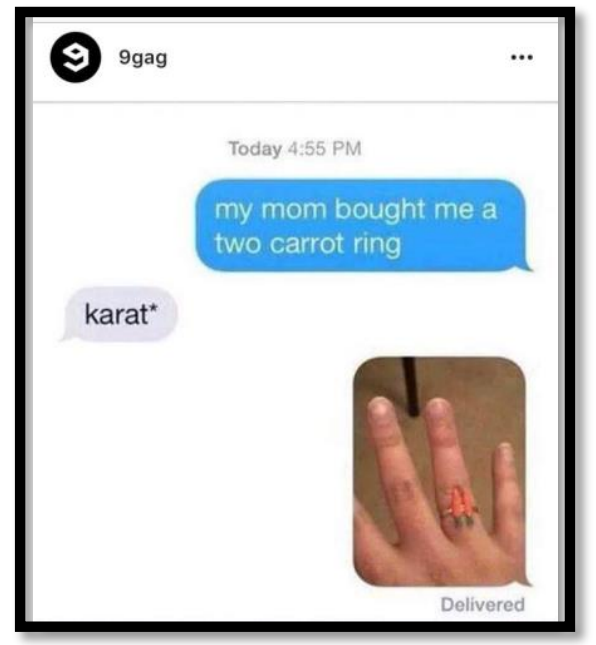

The picture above is a screenshot of a conversation. It can be seen that in the picture there are indications of time are: Today 4:55 PM. Then the conversation which is in a double blue checklist is a sign that it was sent by the sender of text messages and conversations which is in a gray is a reply from the recipient of the message. The sender wrote that his mother bought him a ring with (garnish) 2 carrots (carrots two rings).

After reading the message, the message recipient to assume that there has been a writing mistake or typo by the sender of the message, as shown by a reply that said 'rust * ", there is a possibility that the intended recipient of the message was largely that the ring provided by the sender of the message is equal to 2 carats. Where carat is a unit to declare the gold content. But at after a reply to the sender by sending her picture was wearing a ring with a decorative 2 carrots 
(carrots). It can be concluded that what is meant by the sender of the message with "two carrots rings" is really a ring that is decorated with replicas that resemble 2 carrots (carrots).

Picture 2. A Meme of Difference Between Mom and Dad in Worrying Their Kid

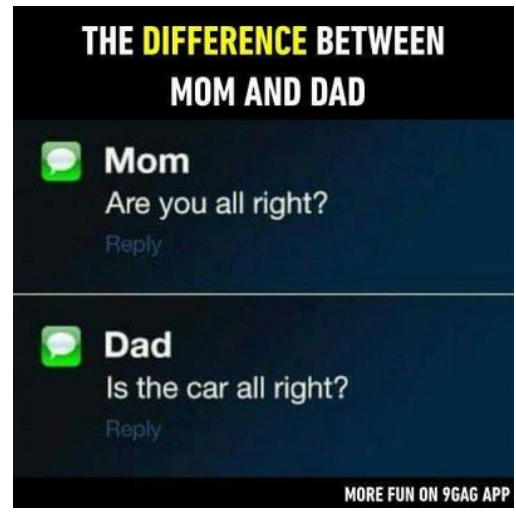

The picture shows about a boy who received a message from two people, namely: a message from the mother and father. It can be seen that the screenshots shown are pages in the cell phone notification (notification center). By using multimodality method of analysis, it will be explained what the purpose of the image. The picture is as follows:

From both messages received it can be assumed that the message recipient is a child who brings a vehicle is a car. It can be seen from the word "car" so that it can be seen that a child driving a car and may have an accident. Furthermore it can be assumed that the child suffered an accident. It can be seen from the words in the message sent by his mother "are you all right?'

The difference of the second message is that the mother is more focused to inquire and find out how his situation, if he was okay or not. This tends to focus more to ask and find out how the car driven state of the child, okay or not. That is indirectly explained that she was very concerned about the state of his son, while the father was more careful to the safety and the state of the car compared with his own safety.

Picture 3. The Conversation about A Man Sleeping After Test

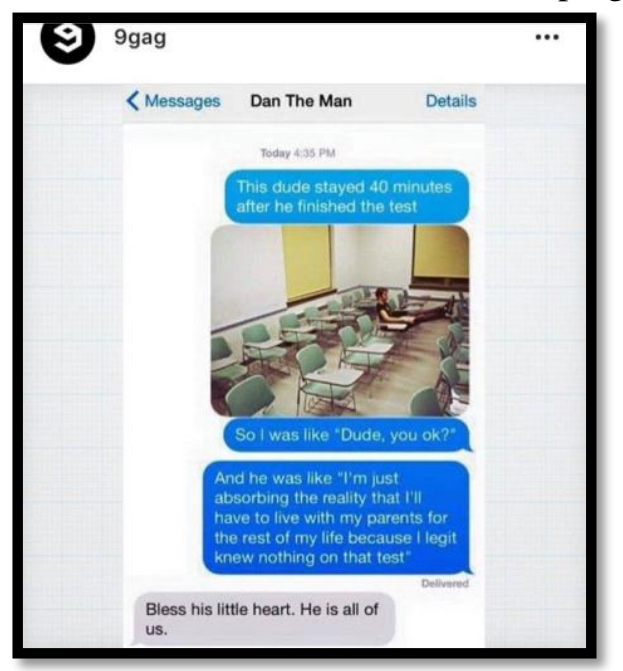

The picture above is a screenshot of a conversation between two friends who were talking about the others. Color blue conversation is the text sent by the sender and gray text is the text of a reply from the recipient of the message. The sender of a message telling that the classmate named Dan silent in the classroom for 40 minutes after finished working on a test. It can be seen that the sender of the message also send pictures or photos that are sitting samba and straighten his legs to a chair, and he was like brooding about something. 
Then the messenger told me again that he had asked Dan why he just remains silent after the test. Then Dan replied "I just realized that I have to live with my parents during my life, because at the time of a test answer was he really does not know anything". It can be assume that and feel like a failure in his exam, there is the possibility that Dan is a lazy boy or rarely learn or study his exam, that he regrets not being able to answer questions on the test and be aware and concerned that later he could not succeed because he will forever stay with his parents, in other words Dan is pessimistic.

Furthermore, the recipient reply to "bless his heart" can be assumed that the receiver was flattered to the answer and that in fact they all (classmates or students) also had anxiety the same, that fear may not count on to answer the questions in that test that they are afraid will fail either.

Picture 4. The Reply in Twitter

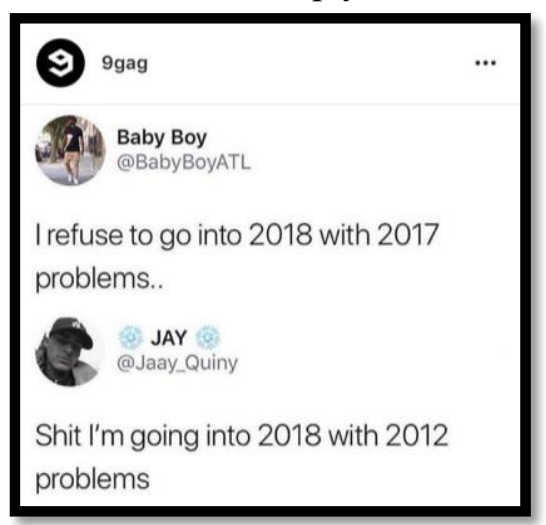

This image is a conversation or mention and reply is in the features of Twitter. Where there is a conversation between two participants. There is participants makes nudge on twitter and replying to nudge the participants. The meme is as follows:

The sender of a message saying that "I refused (to enter) in 2018 to (bring) the problem (which I have) in the year 217". Based on this sentence can be assumed that the message sender does not want to enter 2017 with the troubles in 2017 remain unresolved. While the message recipient reply "S *** $\mathrm{t}$ ! I entered 2018 with the problems that exist in the year 2012 ". Based on this sentence we can assume that your recipient has a lot of problems in 2012, which makes it necessary to start 2018 incorrectly in 2012 and subsequent years that may be unresolved.

Picture 5. The Conversation between A Mother and A Daughter

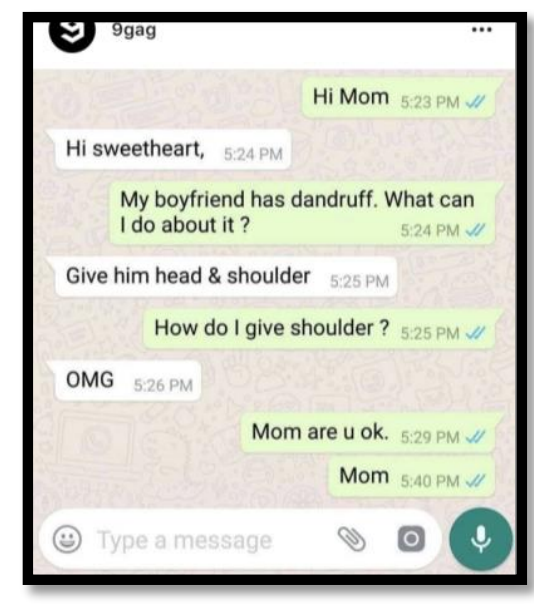

The following figure is a conversation between two participants, namely: a mother and a daughter. The conversation is about the girl who asked for advice to her mother, what he should 
do to help overcome the problem of her boyfriend's dandruff. Then she suggested that gave it a "Head and Shoulder". As we know that Head and Shoulder is an anti-dandruff shampoo brands.

But her daughter did not know what the purpose of "Head and Shoulder'. She really thought that what her mom mean about "Head and Shoulder" is really the shoulders and head, literally. Though the intended mother is a free anti-dandruff shampoo can help override her daughter's boyfriend problems (dandruff). Therefore, in this conversation happened a miscommunication. The mother did not explained that "Head and Shoulder" is a brand of a shampoo, and the girls do not know the information. She also had no idea if his daughter does not understand that the "Head and Shoulder" is a brand of a shampoo. So then the mother responded "OMG" which stands for "Oh My God" and then ignore the other messages sent by her daughter.

Picture 6. The Conversation of An Uber Driver and A Customer

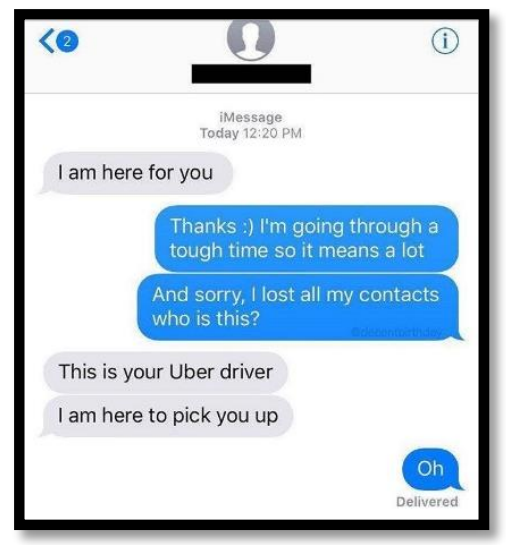

The image is a conversation between two participants is uber drivers and customers. The conversation began when uber driver sends the message "I am here for you", may be referred by uber driver that the message is to tell customers that he is ready to pick her up. As it is known that the uber driver is an online-based transportation services.

But there is a misunderstanding, the customers of the uber think that, everyone who sends the message is someone who she really know in person and care about her, so she thought that it must be someone who cares, but the customers uber, tell out the problem to the sender of the message, because she does not know who sent the message because she lost all of her contacts in her phone. Therefore she asked "who is this?" the uber driver answered "This is your uber driver" that the uber driver answered "I am here" where it actually means that the uber driver has arrived to pick up the customers. Not to give encouragement or some emotional spirit to the customers who are experiencing problems.

Picture 7. The Meme of A Man in A Quiz

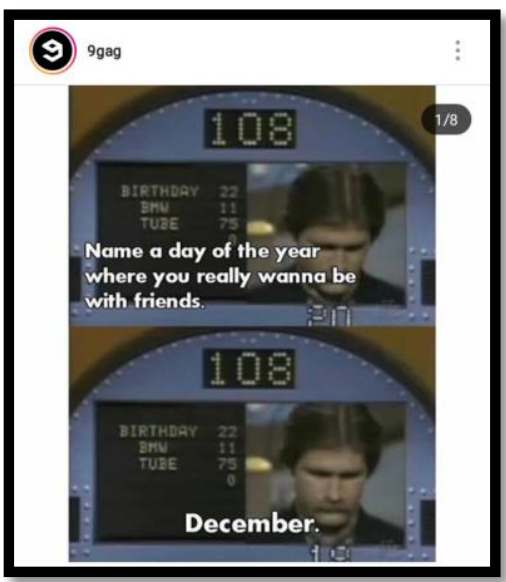


The picture above is a conversation between two people who participated. That is hosted in a quiz word and a man who participated. In a quiz usually a host will ask some specific questions that must be answered by the participants. In this game show, the host asked the participants "mentioned a day of the year you really want to spend time together with friends" then the man answered "December".

As we know that December is not a name of days but it's the name of month. Based on the answers given by the participants, the man can be seen that the reason he answered December which is not the name of day, it comes to a possibility that the participant is a antisocial or the type of person who loner, so he does not have a single day of the year that he wants spend or pass with his friends.

The following image is a screenshot of a conversation between two friends. Green conversation sign is a conversation or text that is sent by the sender of the message while the conversation or text is gray text is a reply from the recipient of the message. The image of the screenshot on the conversation is as follows:

Picture 8. The Conversation of Answering Chat with Chemical Elements

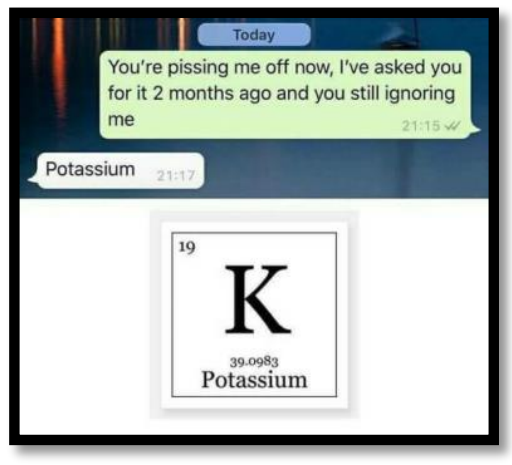

According to picture, it can be seen that the sender of the message was irritated this can be proved by the words "you are pissing me off now" because he asked something to the recipient of messages that can be proved with the phrase "I've asked you for it two months ago" in this context is not explained in detail what are called into question by the sender, because he just mentioning briefly by using the word "it", then the sender of the message was that the recipient has ignored it for months, it is evident from the phrase ".. 2 months ago "and the phrase "you still ignoring me" means that the sender of the message remains neglected or ignored by the recipient of the message. But the response or feedback given by the message recipient in the form of a reply brief is only one word "Potassium".

As noted in the image that Potassium is a chemical element with the symbol of the letter "K". Letter $\mathrm{K}$ itself in the slang words are often interpreted as an abbreviation of the word "OK", which means "Yes" or "OK". Based on this analysis it can be seen that indeed the recipient of the message indirectly is completely ignoring the sender of the message, because when the sender sends the message that angry, but the message recipient still responded with a sense of no matter.

Picture 9. The Conversation of Asking to Hang Out

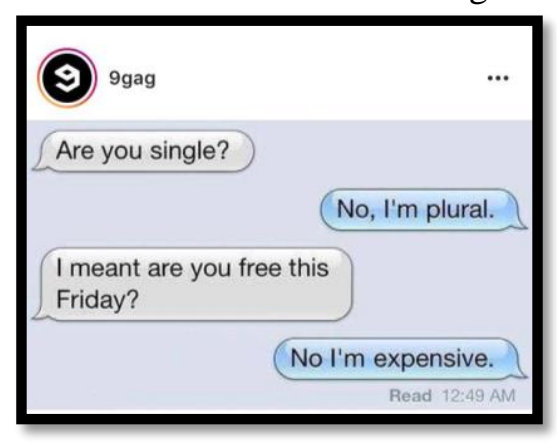


The image above is a screenshot of a conversation between two people. However, based on these conversations cannot be clearly identified about the gender of each participant. So based on that conversation can be identified only by the sender and the recipient. Sender messages are marked with gray or conversation later called $\mathrm{X}$, and the recipient is marked with a blue or conversation later called $\mathrm{Y}$. (since $\mathrm{X}$ is the first who sent a message). In pictures also show details of the time when the message was sent and returned by each participant.

Based on these images can be seen that $\mathrm{X}$ asked $\mathrm{Y}$ if he was single in the sense of having a lover, a date or not. It can be shown to be the phrase "are you single?'. Then Y reply "No, I'm plural". Answer Y is the opposite or reverse of the word delivered by X, where the opposite of a Single (alone or single) is plural. Responding to serve targeted, then X clarify the intent of the question is that by replying "I meant are you free this Friday? "which means X ensures back and asked if Y "free" (no events on this Friday).

Based on this sentence can be assumed that the $\mathrm{X}$ ensures $\mathrm{Y}$ have a date or not because $\mathrm{X}$ would like to invite $\mathrm{Y}$ go on a date on Friday. But this time the $\mathrm{Y}$ remains reply text with the opposite words from the message sent by Y. X responded "no. I'm expensive". As where we know that the opposite of a free (gratis literally) is expensive (expensive). So based on the results of the analysis can be acknowledge that $\mathrm{X}$ would like to invite $\mathrm{Y}$ to date but $\mathrm{Y}$ does not understand the message that is sent to him or is likely a smooth way for $\mathrm{Y}$ to refuse a date from $\mathrm{X}$.

The following image is a screenshot of a conversation between two people. However, based on these conversations can not be clearly identified gender of each participant. So based on that conversation can be identified only by the sender and the recipient. Sender messages are marked with gray or conversation later called $\mathrm{X}$, and the recipient is marked with a blue or conversation later called $\mathrm{Y}$. (since $\mathrm{X}$ first sending a message). In pictures also show details of the time when the message was sent and returned by each participant. The screenshot of the conversation is as follows:

Picture 10. The Conversation of Expressing Feelings in Chat

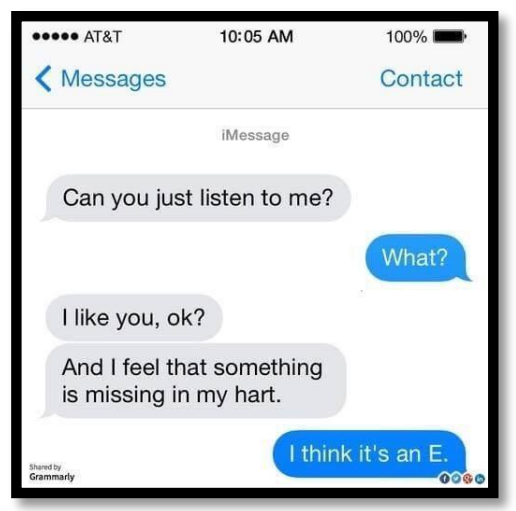

$\mathrm{X}$ sends a message to $\mathrm{Y}$ by saying "Can you listen to me?" And $\mathrm{Y}$ reply to "what". Can be in the know that $\mathrm{X}$ wanted to tell something to the $\mathrm{Y}$ and he asks $\mathrm{Y}$ to listen or respond to $\mathrm{Y}$ to answer "what". Then $\mathrm{X}$ retaliated and told me he liked $\mathrm{X}$ and now he felt that like there is something missing in his heart. This can be evidenced by the phrase "I like you, ok?" And "and I feel that something is missing in my heart." There is a possibility that the definition of something missing in his heart was that he felt homesick or more to the $\mathrm{Y}$, which is why $\mathrm{X}$ honestly say that she likes Y. But Y feedback given is that the Y really tell what the "something missing" is, instead responds honesty expressed by $\mathrm{X}, \mathrm{Y}$ justify grammar precisely because $\mathrm{X}$ has an error typing or typo. Where $\mathrm{X}$ wrote "hart" which probably means the "heart" and X corrected the grammar by reply to the message "I think it is an E". That means he gives the answer or feel that something is missing it is the letter E. Based on the meme, it can be assumed that X likes $\mathrm{Y}$. While the $\mathrm{Y}$ is not like $\mathrm{X}$ because it ignores the $\mathrm{Y}$ or $\mathrm{X}$ does just focus on writing error or typo made by $\mathrm{X}$ (in accidentally). 
This image below is a conversation or mention and reply is in the features of Twitter. Where there is a conversation between two participants. Namely a participant who makes tweet on twitter and replying to the another participants. The screenshot of the conversation is as follows:

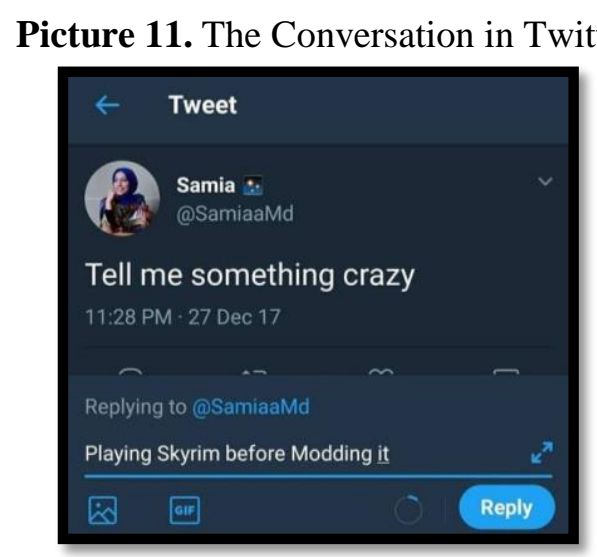

From the figure can be seen the time details when the messages was sent or updated. In the picture, we an also see the profile of both participants. A photo belongs to a woman named Samia. The woman wrote "tell me something creazy" it means that she wanted to hear from other people about something that she considered "crazy". The word "crazy" here can mean something great, good or astonishing. Then there were someone who replied the message by saying "Playing Skyrim before modding it". As we know that Skyrim is a game on personal computers known as quite a difficult game to play,

Based on these images, perhaps you can understand the meaning that it is only a certain group of gamers who will understand the message. Because they probably understand better about how to play the game Skyrim, especially if it is known that the game Skyrim is one of the game that is quite difficult to play, so if you play the game without modding it, than the gamers would consider it as "something crazy".

The following image is a screenshot of a conversation between two people. However, based on these conversations can not be clearly identified gender of each participant. So based on that conversation can be identified only by the sender and the recipient. Sender messages are marked with a blue or conversation later called $X$, and the recipient is marked with gray or conversation later called $\mathrm{Y}$. (since $\mathrm{X}$ is the first who sent the message). In this pictures also show details of the time when the message was sent and returned by each participant. The screenshot of the conversation is as follows:

Picture 12. The Conversation about Exam in Whatsapp

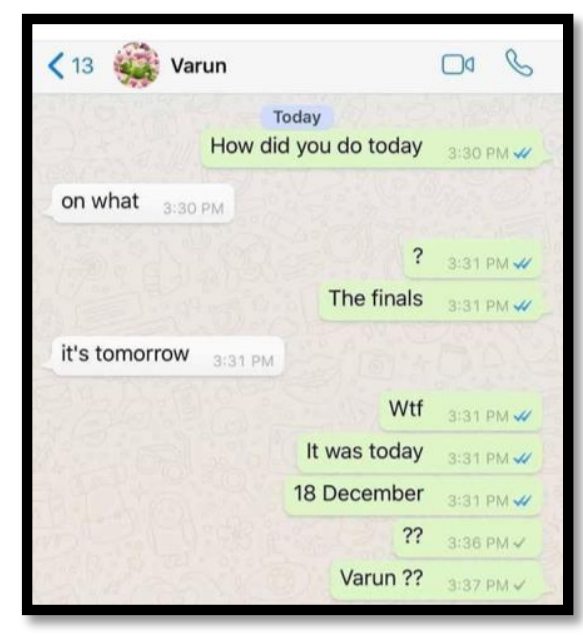


In the picture $\mathrm{X}$ asks Varun or $\mathrm{Y}$ "how did you do today" but $\mathrm{X}$ does not clearly mention what it is doing so specifically that $\mathrm{Y}$ replied to the message with "on what" meaning $\mathrm{Y}$ also asked specifically what it meant by $\mathrm{X}$. Then $\mathrm{X}$ responds with the sentence "?" And "the finals". The reply message sent by X said question mark "?" Is indirectly showing the wonder why Y does not remember the finals at all. "The finals" itself can mean a test. Then Y replies that the test will actually be done tomorrow, this can be proved by the phrase "it's tomorrow".

But then X feel surprised by her reply "WTF" and tell Varun that the examination is on the 18th of December. Based on these images can be assumed that Varun or Y forgotten when the test was carried out, which is why he responded that the examinations is out tomorrow or it means December 19, when the exam is actually on 18 December. $X$ and $Y$ want to know how complete the test. Y no longer reply to a message from X. It can be seen from the double symbol checklist in the picture, which means the message is received by the recipient of the message but have not yet read.

\section{Conclusion and Suggestion}

In this sub-chapter, the writer presents the outcome of the study according to the language errors analysis as follow:

1. According to the result of the analysis meme by using Multimodality Analysis method, the writer concluded that meme is capable of being a medium to deliver a message and entertainment nowadays. Because meme has been accepted by the worldwide internet user.

2. According to the result of the analysis meme by using Multimodality Analysis method, it can also know that nowadays meme has become a massive communication medium, which accepted by the internet users globally, to deliver a message where the message or the entertainment itself can be a sign of a meaning to a certain message. Meme can also become a humor in a form of visual image, which also combine text and image.

Then, the writer presents the advice to suggest improvements for the future writing product the as follow:

1. To analyses many visual image such as meme by using Multimodality Analysis. Therefore, it can be able to help people interpreting the meaning of the meme itself.

2. It would be better for the reader to acknowledge the whole meaning of the meme. Therefore the humor or entertainment aspects can be understood without any ambiguity.

\section{References}

Chen, Y. 2010. Exploring dialogic engagement with readers in multimodal EFL textbooks in China. Visual Communication, 9(4), 485-506.

Dawkins, R. 1976. The selfish gene. New York: Oxford University Press

Iedema, R. 2003. Multimodality, resemuitization: Extending the analysis of discourse as multisemiotic practice. Visual communication, 2(1), 29-57.

Keraff, G. 2010. Diksi dan gaya bahasa. Jakarta: PT. Gramedia Pustaka Utama.

Kreidler, C. W. 1998. Report of the sixteenth annual round table meeting on linguistics and language studies. Washington: Georgetown University Press.

Kress, G., \& Van Leeuwen, T. 2006. Reading images: The grammar of visual design. New York: Routlege.

Saeed, J. 1997. Semantics. UK:Blacwell Publisher Ltd.

Diaz, C. M. C. 2013. Defining \& characterizing the concept of internet meme. Copenhagen; University of Copenhagen.

Tarigan, H. G. 2009. Pengajaran gaya bahasa. Bandung: Penerbit Angkasa. 